\title{
EHF SOLID STATE TRANSMITTERS FOR SATELLITE COMMUNICATIONS
}

\author{
Jorg E. Raue \\ TRW Electronic Systems Group \\ One Space Park \\ Redondo Beach, California 90278
}

\begin{abstract}
During the past three years, interest in satellite communications in the frequency bands above Ku-band has expanded dramatically. As a result, a number of key technology developments, targeted to meet specific next generation spaceborne needs, were undertaken. The state-of-the-art in solid state power transmitters, including critical passive component technology, is presented. This includes filters as well as a series of rugged high performance ferrite components such as isolators, circulators and latching switches.
\end{abstract}

\section{SUMMARY}

In the solid state power transmitter area, several key development efforts are summarized in Table 1.

\section{Table 1. Summary of Solid State Power Transmitter Developments for Space Applications}

\begin{tabular}{|c|c|c|c|}
\hline Type & Frequency & Power Output & Comment/Status \\
\hline FET Amplifier & $15 \mathrm{GHz}$ & 7 Watts & $\begin{array}{l}1 \mathrm{GHz} \text { BW. Complete and space } \\
\text { qualified (TRW) }\end{array}$ \\
\hline FET Amplifier & $20 \mathrm{GHz}$ & 8 Watts & $\begin{array}{l}\text { Eight } 1.25 \mathrm{~W} \text { modules. } 8 \mathrm{~W} \\
\text { achieved March } 1983\end{array}$ \\
\hline IMPATT Amplifier & $20 \mathrm{GHz}$ & 20 Watts & $\begin{array}{l}19 \text { Watts achieved, gain } 10 \mathrm{~dB} \text {, } \\
100 \mathrm{MHz} \mathrm{BW} \text { (TRW) }\end{array}$ \\
\hline IMPATT Amplifier & $38 \mathrm{GHz}$ & 5 Watts & $\begin{array}{l}10 \mathrm{~dB} \text { gain, } 500 \mathrm{MHz} \text { BW. } 8 \% \text { ef- } \\
\text { ficiency. Completed } 1979 \text { (TRW) }\end{array}$ \\
\hline IMPATT Amplifier & $41 \mathrm{GHz}$ & 11 Watts & $\begin{array}{l}30 \mathrm{~dB} \text { gain, } 400 \mathrm{MHz} \text { BW, } 7 \% \text { effi- } \\
\text { ciency. Completed } 1981 \text { (TRW) }\end{array}$ \\
\hline
\end{tabular}



IMPATT Amplifier
$44 \mathrm{GHz}$
0.5 Watts
$2 \mathrm{GHz}$ BW. First 6 stages, with $40 \mathrm{~dB}$ gain and $0.5 \mathrm{~W}$ output power completed June 1982 (TRW)
IMPATT Amplifier
$44 \mathrm{GHz}$
5 Watts
$2 \mathrm{GHz}$ BW. Status: 3.5 Watts achieved.

Each of the above amplifiers will be described in detail, three of them are shown in Figures 1 to 3 below.

Since 1975, a series of ferrite high performance components have been developed at TRW. The rugged design employed in both circulators and switches was readily space qualifiable and has been selected and used on a number of space programs. Table 2 and Figure 4 summarize this hardware.

\section{Table 2. Typical Performance of Space Qualified High Power Ferrite Components}

\begin{tabular}{|c|c|c|c|c|c|c|}
\hline $\begin{array}{c}\text { Frequency } \\
\text { Range } \\
\text { (Ghz) } \\
\end{array}$ & Type & $\begin{array}{c}\text { Insertion } \\
\text { Loss } \\
(\mathrm{dB}) \\
\end{array}$ & $\begin{array}{c}\text { Isolation } \\
(\mathrm{dB}) \\
\end{array}$ & $\underline{\text { VSWR }}$ & $\begin{array}{c}\text { Bandwidth } \\
(\%)\end{array}$ & $\begin{array}{l}\text { CW Power } \\
\text { (Watts) }\end{array}$ \\
\hline $7-10$ & Circulator & 0.1 & -25 & 1.10 & 35 & 100 \\
\hline $7-8.5$ & Latching Switch* & $0.35^{*}$ & $-37 *$ & 1.05 & 3 & 100 \\
\hline $18-23$ & Circulator & 0.2 & -25 & 1.2 & 24 & 75 \\
\hline $17.5-20.5$ & Latching Switch* & 0.3 & -20 & 1.2 & 15 & 75 \\
\hline $27.5-30$ & Latching Switch* & $0.4^{*}$ & $-40 *$ & 1.2 & 9 & 6 \\
\hline $26.5-34$ & Circulator & 0.1 & -20 & 1.2 & 26 & 12 \\
\hline $32-40$ & Circulator & 0.1 & -20 & 1.2 & 23 & 12 \\
\hline $37-46$ & Circulator & 0.15 & -20 & 1.2 & 22 & 12 \\
\hline $59-62$ & Circulator & 0.3 & -20 & 1.25 & 3 & $* *$ \\
\hline $92-99$ & Circulator & 0.5 & -20 & 1.3 & 7 & $* *$ \\
\hline
\end{tabular}

In the filter area, we have evaluated a number of different transmission media in constructing filters, but have found only waveguide filters to be acceptable for demanding space applications. A comparison of the different types of filters evaluated is shown in Figure 5. 

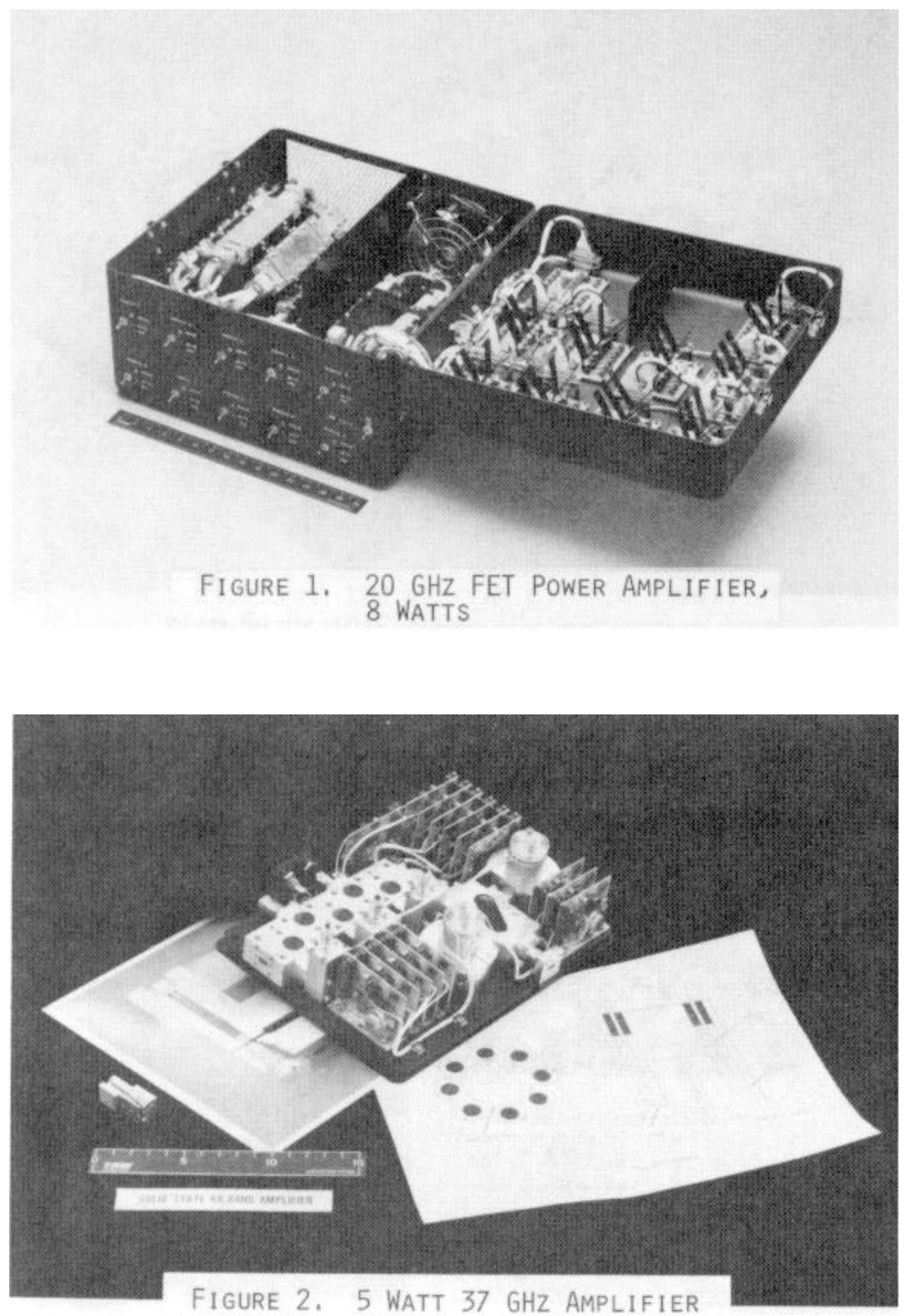


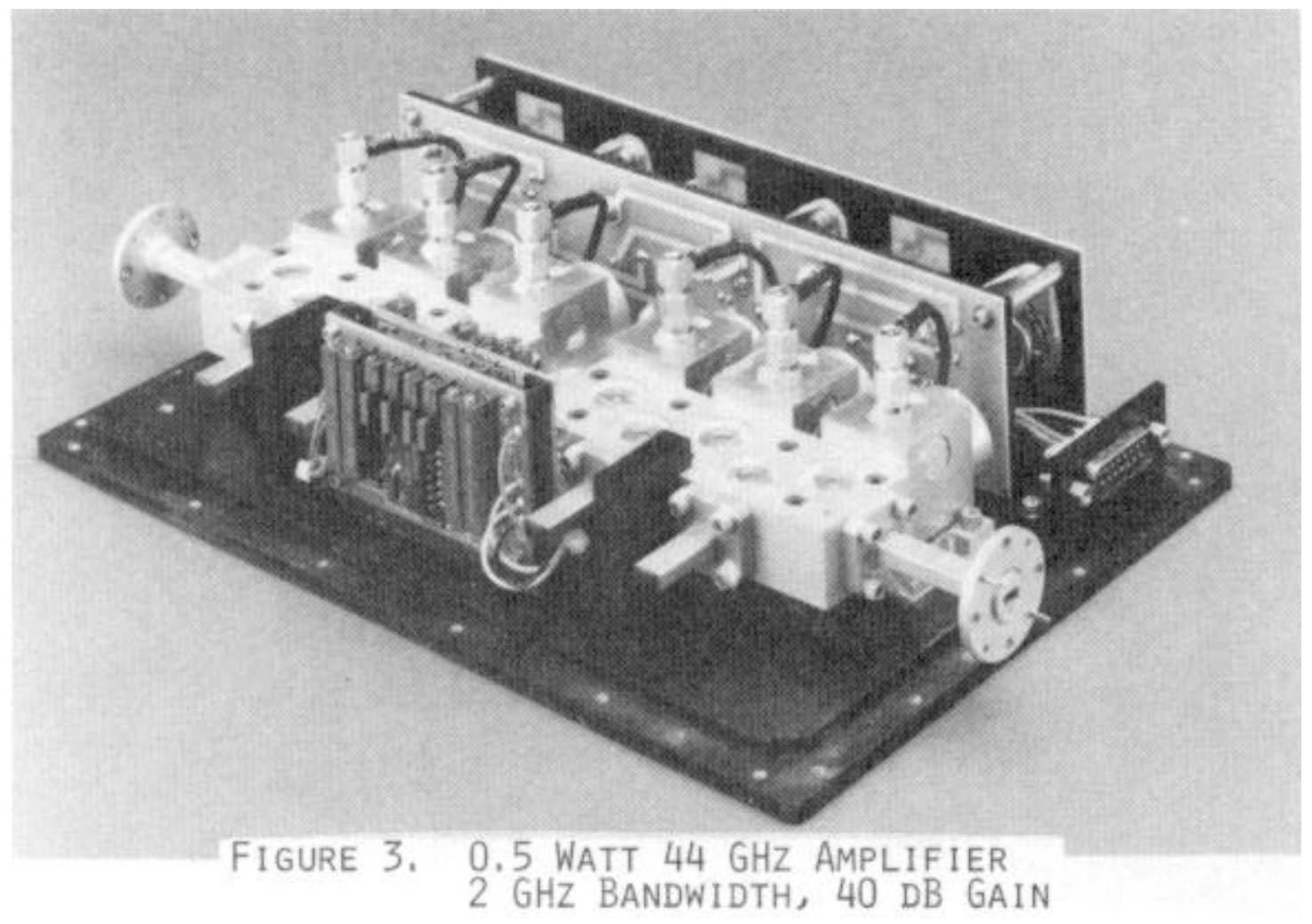

TRW

FIGURE 4 , LOW LOSS BROADBAND CIRCULATORS

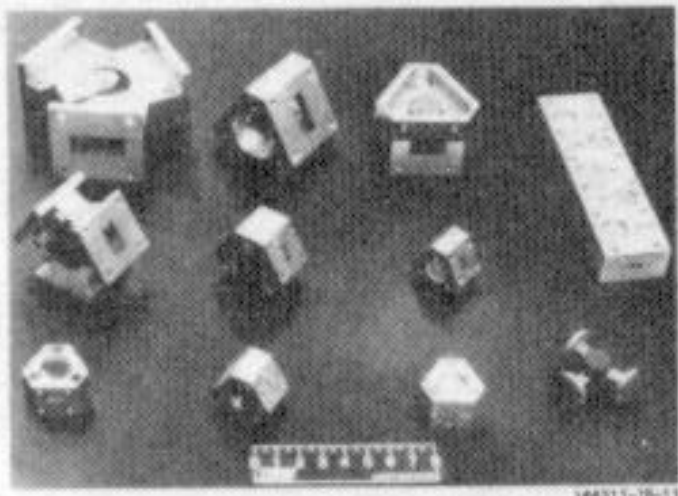

\section{FEATURES}

- nOVEl CMLINoRical junction oesicin

- Low loss Widegand

- LOW VSWR, MGGH ISOLATION

- the ramally stagle

- simple, structuaali r aubeed

- ONe to five Junctions

- no inter-stade ma tChing requireo

- OESION SELL. HIOEXING - NO EPOXIES OA ADHESIVES

- DESICN PROVEN 7 YO $47 \mathrm{GH}$

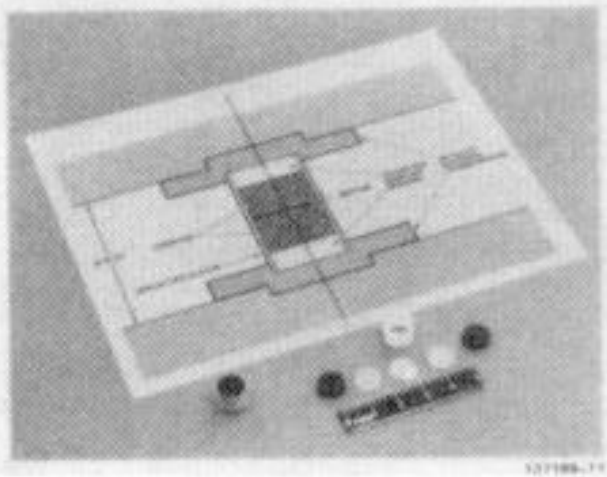




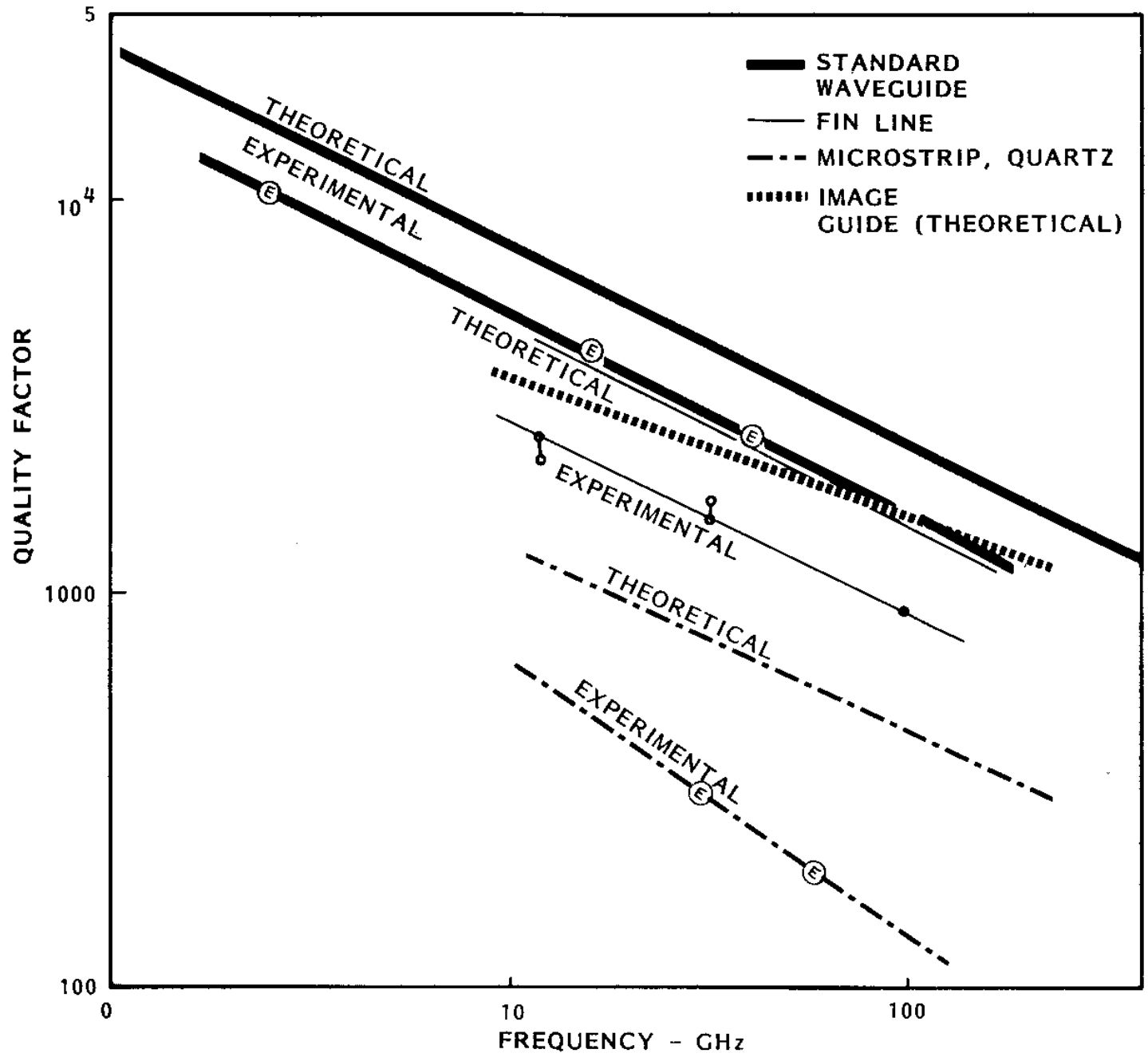

FIGURE 5. THEORETICAL vS. EXPERIMENTAL TRANSMISSION RESONATOR (FILTER) FOR VARIOUS TRANSMISSION MEDIA (FUNDAMENTAL MODE) 